PROCEEDINGS OF THE

AMERICAN MATHEMATICAL SOCIETY

Volume 129, Number 4, Pages 1041-1049

S 0002-9939(00)05688-4

Article electronically published on October 4, 2000

\title{
MORITA EQUIVALENCE AND PEDERSEN IDEALS
}

\author{
PERE ARA
}

(Communicated by David R. Larson)

\begin{abstract}
We show that two $C^{*}$-algebras are strongly Morita equivalent if and only if their Pedersen ideals are Morita equivalent as rings with involution.
\end{abstract}

\section{INTRODUCTION}

Two rings with identity $R$ and $S$ are said to be Morita equivalent in case their categories of unital right modules are equivalent. The classical Morita Theorems give the precise conditions under which two unital rings are Morita equivalent (see 2] for example). It follows from these theorems that two unital rings $R$ and $S$ are Morita equivalent if and only if there is an $S-R$-bimodule $P$ such that $P$ is a finitely generated projective generator both as a left $S$-module and as a right $R$-module. For $C^{*}$-algebras, there is a related notion, introduced by Rieffel [19], 20], which is formally close to that characterization. Namely, two $C^{*}$-algebras $A$ and $B$ are said to be strongly Morita equivalent in case there is a full Hilbert $B-A$ bimodule (see Section 2 for the precise definition). It is important to remark that the definition for $C^{*}$-algebras does not make the assumption that the algebras have identity. Moreover, the theory is especially useful in the non-unital case. Also, the Hilbert module is not assumed to be finitely generated or projective. In fact, it turns out that the fact of $E$ being finitely generated projective is a special feature of the unital case, in the sense that it is automatically satisfied if our $C^{*}$-algebras are unital, but it is not necessarily true in the general case. This was observed by Rieffel [21, Proposition].

It was proved by Beer that two unital $C^{*}$-algebras are strongly Morita equivalent if and only if they are Morita equivalent as rings [5 Theorem 1.8]. Our aim here is to generalize this result to the non-unital situation. For this, we need a concept of Morita equivalence for non-unital rings, at least for special classes of them. There are many papers dealing with this topic (see for example [1], [3, [9], [12, [15, [16]). The theory works in a satisfactory way for idempotent non-degenerate rings. Here, a ring $R$ is said to be idempotent if $R=R^{2}$; that is, $R$ is linearly spanned by the elements of the form $r_{1} r_{2}$, with $r_{i} \in R$, and $R$ is non-degenerate in case, for $x \in R, x R=0$ or $R x=0$ implies $x=0$. For an idempotent, non-degenerate $\operatorname{ring} R$, we consider the category $\operatorname{Mod}-R$, which is the full subcategory of the category of all the right $R$-modules, whose objects are the modules $M$ such that

Received by the editors March 30, 1998 and, in revised form, June 25, 1999

2000 Mathematics Subject Classification. Primary 46L08, 16 D90.

This work was partially supported by DGYCIT and the Comissionat per Universitats i Recerca de la Generalitat de Catalunya. 
$M=M R$ (that is, $M$ is spanned by the elements of the form $m r$, with $m \in M$ and $r \in R)$ and $M$ is non-degenerate in the sense that $m R=0$ implies $m=0$. Note that $\operatorname{Mod}-R$ is the usual category of unital right $R$-modules in case $R$ is a unital ring. The category $\operatorname{Mod}-R$ is a Grothendieck category; that is, a cocomplete abelian category such that direct limits are exact and has generators. Thanks to the nice properties of $M o d-R$, suitable versions of the Morita Theorems are true for idempotent non-degenerate rings (see [9, [16]).

It turns out that the appropriate category of modules to look at when $A$ is a $C^{*}$-algebra is not the category $M o d-A$, but the category $M o d-K_{A}$, where $K_{A}$ is the Pedersen ideal of $A$, i.e., the minimal dense ideal of $A$. Our main result is that two $C^{*}$-algebras are strongly Morita equivalent if and only if the categories Mod $-K_{A}$ and $M o d-K_{B}$ are equivalent (through an equivalence which is well behaved with respect to the involutions). In order to prove this, we develop some basic facts about the Pedersen submodule of a Hilbert $C^{*}$-module is Section 1. The main point in working with Pedersen ideals and Pedersen submodules is that we don't need to take closures in the usual Hilbert module constructions, and this enables us to apply directly the algebraic theory.

After completing this work, I have received a preprint from David Blecher [6], containing a characterization of strong Morita equivalence by using operator modules. His methods are completely different from ours.

\section{The Pedersen submodule of a Hilbert $C^{*}$-module}

In this Section we develop the basic properties of the Pedersen submodule of a Hilbert $C^{*}$-module. This is a generalization of the Pedersen ideal of a $C^{*}$-algebra $A$ in that the Pedersen submodule of the Hilbert $A$-module $A$ is precisely the Pedersen ideal of $A$.

Definition 1.1. Let $A$ be a pre- $C^{*}$-algebra. An inner-product $A$-module is a linear complex space $E$ which is a right $A$-module, together with a map $(x, y) \mapsto\langle x, y\rangle$ : $E \times E \rightarrow A$ such that, for $x, y, z \in E, \alpha, \beta \in \mathbb{C}$, and $a \in A$, we have

(i) $\langle x, \alpha y+\beta z\rangle=\alpha\langle x, y\rangle+\beta\langle x, z\rangle$,

(ii) $\langle x, y a\rangle=\langle x, y\rangle a$,

(iii) $\langle y, x\rangle=\langle x, y\rangle^{*}$,

(iv) $\langle x, x\rangle \geq 0$; if $\langle x, x\rangle=0$, then $x=0$.

If, in addition, $A$ is a $C^{*}$-algebra, and $E$ is complete with respect to the norm given by $\|x\|^{2}=\|\langle x, x\rangle\|$, then $E$ is said to be a Hilbert $A$-module. A Hilbert $A$-module is said to be full if the ideal $\langle E, E\rangle$ is dense in $A$.

Given an inner-product $A$-module over a pre- $C^{*}$-algebra $A$, we can complete $E$ to get a Hilbert $\bar{A}$-module (see [13, p. 4]). This Hilbert $\bar{A}$-module will be denoted by $\bar{E}$.

We denote the $*$-algebra of adjointable operators on an inner-product $A$-module $E$ by $\mathcal{L}_{A}(E)$. For $x, y \in E$, denote by $\theta_{x, y}$ the map from $E$ to $E$ given by $\theta_{x, y}(z)=$ $x\langle y, z\rangle$. Then $\theta_{x, y} \in \mathcal{L}_{A}(E)$, with $\theta_{x, y}^{*}=\theta_{y, x}$. Let $\mathcal{F}_{A}(E)$ be the linear span of $\left\{\theta_{x, y} \mid x, y \in E\right\}$. Then $\mathcal{F}_{A}(E)$ is a (two-sided) ideal of $\mathcal{L}_{A}(E)$. If $A$ is a $C^{*}$-algebra and $E$ is a Hilbert $A$-module, we denote the norm-closure of $\mathcal{F}_{A}(E)$ by $\mathcal{K}_{A}(E)$ (see [13, p. 10]).

For a $C^{*}$-algebra $A$, we denote the Pedersen ideal of $A$ by $K_{A}$ (see [17, 5.6]). Recall that $K_{A}$ is the minimal dense ideal of $A$. If $A=C_{0}(X)$ for a locally compact 
Hausdorff space $X$, then $K_{A}=C_{c}(X)$, the algebra of continuous functions on $X$ with compact support.

Definition 1.2. Let $A$ be a $C^{*}$-algebra and let $E$ be a Hilbert $A$-module. The Pedersen submodule of $E$ is $P_{E}=E K$, where $K$ is the Pedersen ideal of $\overline{\langle E, E\rangle}$.

Note that $\left\langle P_{E}, P_{E}\right\rangle=K\langle E, E\rangle K=K$, so $E\left\langle P_{E}, P_{E}\right\rangle=E K=P_{E}$. Here is a useful description of the Pedersen submodule.

Proposition 1.3. Let $A$ be a $C^{*}$-algebra and let $E$ be a Hilbert $A$-module. Let $K$ be the Pedersen ideal of $\overline{\langle E, E\rangle}$. Then

$$
P_{E}=\{e \in E \mid\langle e, e\rangle \in K\} .
$$

Proof. Assume that $e \in E K=P_{E}$. Then $e=\sum_{i=1}^{n} e_{i} k_{i}$ for some $e_{i} \in E$ and $k_{i} \in K$. Since $\langle e, e\rangle=\sum_{i, j} k_{i}^{*}\left\langle e_{i}, e_{j}\right\rangle k_{j}$, we see that $\langle e, e\rangle \in K$.

Conversely, assume that $e \in E$ satisfies $\langle e, e\rangle \in K$. Then $|e|=\langle e, e\rangle^{1 / 2} \in K$, and $|e|^{\alpha} \in K$ for all $0<\alpha<1$ (see [17, 5.6.2]). By [13, Lemma 4.4], for each $\alpha$ with $0<\alpha<1$, there is $w \in E$ such that $e=w|e|^{\alpha}$. Consequently, $e \in E K$.

Remark 1.4. By the proof of Proposition 1.3, we have $P_{E}=\{e k \mid e \in E, k \in K\}$.

Proposition 1.5. Let $A$ be a $C^{*}$-algebra and let $E$ be a Hilbert $A$-module. Then $P_{E}$ is the minimal dense $\mathcal{L}_{A}(E)$-invariant submodule of $E$.

Proof. Write $\mathcal{L}=\mathcal{L}_{A}(E)$. Clearly, $P_{E}=E K$ is $\mathcal{L}$-invariant. Since $E\langle E, E\rangle$ is dense in $E$ ([13 p. 6]), we see that $P_{E}=E K$ is a dense $A$-submodule of $E$. Assume that $E_{0}$ is a dense $\mathcal{L}$-invariant submodule of $E$. We will see that $E\left\langle E_{0}, E_{0}\right\rangle \subseteq E_{0}$. For that it suffices to prove that $x\langle y, z\rangle \in E_{0}$ for $x \in E$ and $y, z \in E_{0}$. But $x\langle y, z\rangle=$ $\theta_{x, y}(z) \in \mathcal{L} E_{0} \subseteq E_{0}$, because $E_{0}$ is $\mathcal{L}$-invariant, and so we get $E\left\langle E_{0}, E_{0}\right\rangle \subseteq E_{0}$. Now $\left\langle E_{0}, E_{0}\right\rangle$ is a dense ideal of $\overline{\langle E, E\rangle}$, and so $K \subseteq\left\langle E_{0}, E_{0}\right\rangle$. It follows that

$$
P_{E}=E K \subseteq E\left\langle E_{0}, E_{0}\right\rangle \subseteq E_{0},
$$

as desired.

Let $E$ be a Hilbert $A$-module over a $C^{*}$-algebra $A$. Our next theorem relates the Pedersen submodule of $E$ with the Pedersen ideal of $\mathcal{K}_{A}(E)$.

Theorem 1.6. Let $A$ be a $C^{*}$-algebra and let $E$ be a Hilbert $A$-module. Let $P_{E}$ be the Pedersen submodule of $E$. Then the Pedersen ideal of $\mathcal{K}_{A}(E)$ is exactly $\mathcal{F}_{A}\left(P_{E}\right)$.

Proof. Write $B=\mathcal{K}_{A}(E)$ and $\mathcal{F}=\mathcal{F}_{A}\left(P_{E}\right)$. Denote by $K_{B}$ the Pedersen ideal of $B$. Since $P_{E}$ is $\mathcal{L}_{A}(E)$-invariant, we see that $\mathcal{F}$ is an ideal of $B$. Since $P_{E}$ is dense in $E$, we get that $\mathcal{F}$ is dense in $B$. It follows that $\mathcal{F}$ contains the minimal dense ideal of $B$; that is, $K_{B} \subseteq \mathcal{F}$.

Now let $E_{0}=K_{B} E$ be the linear span in $E$ of the elements of the form $T(e)$, for $T \in K_{B}$ and $e \in E$. We claim that $E_{0}$ is a dense $\mathcal{L}_{A}(E)$-invariant $A$-submodule of $E$. Since $K_{B}$ is an ideal of $\mathcal{L}_{A}(E)$, it is clear that $E_{0}$ is an $\mathcal{L}_{A}(E)$-invariant submodule of $E$. For $x, y, z \in E$, we have $x\langle y, z\rangle=\theta_{x, y}(z)$. Therefore, the fact that $E\langle E, E\rangle$ is dense in $E$ implies that $B E$ is dense in $E$. Since $K_{B}$ is dense in $B$, we conclude that $E_{0}=K_{B} E$ is dense in $E$. 
Now we get from Proposition 1.5 that $P_{E} \subseteq E_{0}$. Now let $x, y \in P_{E}$. Write $x=\sum_{i=1}^{n} T_{i}\left(e_{i}\right)$ for $T_{i} \in K_{B}$ and $e_{i} \in E$. Then we have

$$
\theta_{x, y}=\sum_{i=1}^{n} \theta_{T_{i}\left(e_{i}\right), y}=\sum_{i=1}^{n} T_{i} \theta_{e_{i}, y} \in K_{B},
$$

because $K_{B}$ is an ideal of $B$. Since $\mathcal{F}$ is the linear span of $\theta_{x, y}$ with $x, y \in P_{E}$, we get $\mathcal{F} \subseteq K_{B}$. Therefore, we conclude that $\mathcal{F}_{A}\left(P_{E}\right)$ is the Pedersen ideal of $\mathcal{K}_{A}(E)$, as desired.

The final result of this Section gives a description of the multiplier algebra of the Pedersen ideal of $\mathcal{K}_{A}(E)$ for every Hilbert $A$-module over a $C^{*}$-algebra $A$. Multipliers of Pedersen ideals have been studied extensively by Lazard and Taylor [14], and, more recently, by N.C. Phillips [18]. We refer to these papers for definitions and basic properties of these $*$-algebras. We will denote the multiplier algebra of the Pedersen ideal $K_{A}$ of a $C^{*}$-algebra $A$ by $\Gamma\left(K_{A}\right)$. Recall that $\Gamma\left(K_{A}\right)$ is a complex unital $*$-algebra, and there is a canonical embedding $\iota: K_{A} \rightarrow \Gamma\left(K_{A}\right)$ which has the following universal property in the category of complex $*$-algebras:

For every complex $*$-algebra $D$ and every injective $*$-homomorphism $\varphi: K_{A} \rightarrow D$ such that $\varphi\left(K_{A}\right)$ is an ideal of $D$, there exists a unique $*$-homomorphism $\psi: D \rightarrow$ $\Gamma\left(K_{A}\right)$ such that $\iota=\psi \circ \varphi$.

P. Green and, independently, G.G. Kasparov proved that, for a Hilbert $A$-module over a $C^{*}$-algebra $A, \mathcal{M}\left(\mathcal{K}_{A}(E)\right)=\mathcal{L}_{A}(E)$ (see [10, Lemma 16], [11, Theorem $1]$ ), where $\mathcal{M}(B)$ denotes the multiplier algebra of a $C^{*}$-algebra $B$. We give an analogous result for the multiplier algebra of the Pedersen ideal of $\mathcal{K}_{A}(E)$.

Proposition 1.7. Let $A$ be a $C^{*}$-algebra and let $E$ be a Hilbert $A$-module. Set $B=\mathcal{K}_{A}(E)$. Then $\Gamma\left(K_{B}\right)=\mathcal{L}_{A}\left(P_{E}\right)$.

Proof. By Theorem 1.5, we have $K_{B}=\mathcal{F}_{A}\left(P_{E}\right)$. Let $\iota: K_{B} \rightarrow \mathcal{L}_{A}\left(P_{E}\right)$ be the inclusion map. In order to prove that $\mathcal{L}_{A}\left(P_{E}\right)$ is the multiplier algebra of $K_{B}$ it suffices to check that $\iota$ satisfies the universal property stated before. So assume that $K_{B}$ is embedded as an $*$-ideal in a complex $*$-algebra $D$. Define a map $\psi$ : $D \rightarrow \mathcal{L}_{A}\left(P_{E}\right)$ by the rule

$$
\psi(d)\left(\sum_{i=1}^{n} \theta_{x_{i}, y_{i}}\left(z_{i}\right)\right)=\sum_{i=1}^{n}\left(d \theta_{x_{i}, y_{i}}\right)\left(z_{i}\right),
$$

where $x_{i}, y_{i}, z_{i} \in P_{E}$. (Here we are using that $P_{E}=P_{E}\left\langle P_{E}, P_{E}\right\rangle$.) We have to prove that $\psi(d)$ is well-defined. So assume that $\sum_{i=1}^{n} \theta_{x_{i}, y_{i}}\left(z_{i}\right)=0$ for some $x_{i}, y_{i}, z_{i} \in$ $P_{E}$. Then, we have $\sum_{i=1}^{n} \theta_{x_{i}, y_{i}} \theta_{z_{i}, u}=0$ for all $u \in P_{E}$, and so $\sum_{i=1}^{n}\left(d \theta_{x_{i}, y_{i}}\right) \theta_{z_{i}, u}=$ 0 , which gives $\left(\sum_{i=1}^{n}\left(d \theta_{x_{i}, y_{i}}\right)\left(z_{i}\right)\right)\left\langle P_{E}, P_{E}\right\rangle=0$, and so $\sum_{i=1}^{n}\left(d \theta_{x_{i}, y_{i}}\right)\left(z_{i}\right)=0$.

Therefore, the map $\iota: K_{B} \rightarrow \mathcal{L}_{A}\left(P_{E}\right)$ satisfies the universal property of the multiplier algebra of $K_{B}$, and we conclude that $\Gamma\left(K_{B}\right)=\mathcal{L}_{A}\left(P_{E}\right)$.

Remark 1.8. Let $A$ be a unital $C^{*}$-algebra and let $E$ be a full Hilbert $A$-module. Set $B=\mathcal{K}_{A}(E)$. By Proposition 1.7, $\Gamma\left(K_{B}\right)=\mathcal{L}_{A}\left(P_{E}\right)=\mathcal{L}_{A}(E)$, and so all the multipliers of $K_{B}$ are bounded.

To illustrate Theorem 1.6 and Proposition 1.7 we compute a concrete example. Let $X$ be a locally compact, Hausdorff space. Take $A=C_{0}(X)$ and $E=$ $H_{A}$, the Hilbert $A$-module of all the sequences $\left(f_{n}\right)$, with $f_{n} \in A$, such that $\sum\left|f_{n}\right|^{2}$ converges in $A$. Let $B=\mathcal{K}_{A}\left(H_{A}\right)$, and note that, by [13, p. 63], we 
have $B \cong A \otimes \mathcal{K}$, where $\mathcal{K}$ denotes the algebra of compact operators on a separable infinite-dimensional Hilbert space. The Pedersen submodule of $E$ will be $P_{E}=\operatorname{alglim}_{U} H_{C_{0}(U)}$, where $U$ range over all the open, relatively compact, $\sigma$ compact subsets of $X$. The Pedersen ideal of $B$ is given by $K_{B}=\mathcal{F}_{A}\left(P_{E}\right)$ and it is easily checked from the above that $K_{B}=\operatorname{alglim}_{U} \mathcal{F}\left(H_{C_{0}(U)}\right)$, where, as before, $U$ ranges over the set of all the open, relatively compact, $\sigma$-compact subsets of $X$. Now, by using [18, Theorem 7], we compute that $\Gamma\left(K_{B}\right)=\varliminf_{Y} \mathcal{M}(C(Y) \otimes \mathcal{K})$, where $Y$ ranges over the set of all the compact subsets of $X$, ordered by inclusion, and the maps in the inverse limit are the maps induced by restriction. Of course a lot of unbounded multipliers can arise in this situation.

\section{Morita equivalence And Pedersen ideals}

By the very definition of the Pedersen submodule of a Hilbert $A$-module, it is clear that we can assume that $E$ is a full Hilbert $A$-module; that is, the closure of $\langle E, E\rangle$ is $A$, just by restricting the action to $\overline{\langle E, E\rangle}$. In that case, the algebra of "compact operators" $\mathcal{K}_{A}(E)$ is a $C^{*}$-algebra which is strongly Morita equivalent to $A$ (see [13], 20]), and moreover $E$ has the structure of a full Hilbert $B-A$-bimodule, where $B=\mathcal{K}_{A}(E)$.

Let us recall the definition of a Hilbert $B-A$-bimodule from [8].

Definition 2.1. Let $A$ and $B$ be two $C^{*}$-algebras and $E$ a complex space and $B-A$-bimodule. Suppose that we have sesqui-linear forms ${ }_{B}\langle\cdot, \cdot\rangle$ and $\langle\cdot, \cdot\rangle_{A}$ so that $E$ is both a Hilbert left $B$-module and a Hilbert right $A$-module and that the forms are related by the equation

$$
{ }_{B}\langle x, y\rangle z=x\langle y, z\rangle_{A}
$$

for all $x, y, z \in E$. Then $E$ is a Hilbert $B-A$-bimodule.

A Hilbert $B-A$-bimodule $E$ is said to be full if $E$ is full both as a left Hilbert $B$-module and as a right Hilbert $A$-module. It is proved in [8, Corollary 1.11] that the two norms $\left\|_{B}\langle x, x\rangle\right\|^{1 / 2}$ and $\left\|\langle x, x\rangle_{A}\right\|^{1 / 2}$ coincide.

Two $C^{*}$-algebras $A$ and $B$ are said to be strongly Morita equivalent in case there is a full Hilbert $B-A$-bimodule $E$. This is equivalent to saying that there exists a full Hilbert right $A$-module $E$ such that $B$ is $*$-isomorphic to $\mathcal{K}_{A}(E)$. We refer the reader to [7, [19, 20], 21] for the basic theory of Morita equivalence for operator algebras. It was proved by Beer [5, Theorem 1.8] that two unital $C^{*}$-algebras $A$ and $B$ are strongly Morita equivalent if and only if they are Morita equivalent as rings. Recall that two rings with identity are Morita equivalent if their categories of unital right modules are equivalent. The natural substitute for the category of all modules over a ring in the case where $A$ is a $C^{*}$-algebra seems to be the category of Hermitian right $A$-modules (see [19], 20]). However, Rieffel observed that the fact that two $C^{*}$-algebras have equivalent categories of Hermitian right modules gives a relation strictly weaker than strong Morita equivalence, even in the case in which both $C^{*}$-algebras are unital (see [20], [5]).

From a categorical point of view, one of the main advantages of the category of unital modules over a unital ring is that it is a Grothendieck category, namely a cocomplete abelian category such that direct limits are exact and has generators [22]. The same is true for appropriate module categories over some non-unital rings. An associative ring $R$ is said to be idempotent if $R=R^{2}$; that is, every element 
$r \in R$ can be written as a finite sum $r=\sum s_{i} t_{i}$ for $s_{i}, t_{i} \in R$. $R$ is said to be nondegenerate in case, for $x \in R, x R=0$ or $R x=0$ implies $x=0$. For an idempotent and non-degenerate ring $R$, the useful category is the category $\operatorname{Mod}-R$ which is the full subcategory of the category of all the right $R$-modules whose objects are the right $R$-modules $M$ such that $M=M R$ and, for $m \in M$, the relation $m R=0$ implies $m=0$ (see [9]). It is known that $M o d-R$ is a Grothendieck category.

For the purposes of the present paper we need only the following characterization of Morita $*$-equivalence, which can be taken as its definition.

Definition 2.2. Let $R$ and $S$ be two non-degenerate idempotent rings with involution. Then $R$ and $S$ are Morita $*$-equivalent if there exist $E \in \operatorname{Mod}-R$ and a map $\langle\cdot, \cdot\rangle: E \times E \rightarrow R$ satisfying the following properties:

(i) $\langle x, y+z\rangle=\langle x, y\rangle+\langle x, z\rangle$ for all $x, y, z \in E$,

(ii) $\langle x, y r\rangle=\langle x, y\rangle r \quad(x, y \in E, r \in R)$,

(iii) $\langle x, y\rangle=\langle y, x\rangle^{*} \quad(x, y \in E)$,

(iv) $\langle x, E\rangle=0$ implies $x=0$,

(v) $\langle E, E\rangle=R$,

(vi) $S$ is $*$-isomorphic to $\mathcal{F}_{A}(E)$.

If $R$ and $S$ are Morita *-equivalent through $E$, then there are inverse category equivalences $F: M o d-R \rightarrow M o d-S$ and $G: M o d-S \rightarrow M o d-R$ defined by $F\left(P_{R}\right)=\mathcal{F}(E, P)$ and $G\left(Q_{S}\right)=\mathcal{F}(\widetilde{E}, Q)$. (Here, $\widetilde{E}$ denotes the $R-S$-bimodule which coincides with $E$ as abelian group, but with module operations given by $\widetilde{x} s=\widetilde{s^{*} x}$ and $r \widetilde{x}=\widetilde{x r^{*}}$ for $x \in E, r \in R$ and $s \in S$.) Moreover if $D$ is a right "inner product" $R$-module (i.e. there is a map $\langle\cdot, \cdot\rangle: D \times D \rightarrow R$ satisfying conditions (i)(iv) of Definition 2.2), then $F(D)$ is a right "inner product" $S$-module in a natural way, and if $f$ is an adjointable map, then $F\left(f^{*}\right)=F(f)^{*}$. Similar properties hold for $G$. It is proved in [4] that any category equivalence $F: \operatorname{Mod}-R \rightarrow \operatorname{Mod}-S$ satisfying the above conditions is induced by a module $E$ as in Definition 2.2 (see [4, Theorem 3.1]).

Recall that the Pedersen ideal of a $C^{*}$-algebra $A$ is denoted by $K_{A}$. Our next lemma shows that the Pedersen submodule $P_{E}$ of the full Hilbert right $A$-module $E_{A}$, as defined in Section 1, is in fact a full inner product $K_{B}-K_{A}$-subbimodule of $E$.

Lemma 2.3. Let $E$ be a full Hilbert $B-A$-bimodule, where $A$ and $B$ are arbitrary $C^{*}$-algebras. Then $P_{E}=K_{B} E=E K_{A}$ and $P_{E}$ is therefore the Pedersen submodule of ${ }_{B} E$. Moreover, the restrictions of the sesqui-linear forms ${ }_{B}\langle\cdot, \cdot\rangle$ and $\langle\cdot, \cdot\rangle_{A}$ to $P_{E}$ give $P_{E}$ a structure of full inner product $K_{B}-K_{A}$-module.

Proof. This is a restatement of results obtained in Section 1. By definition, $P_{E}=$ $E K_{A}$. By the Morita theory of $C^{*}$-algebras, $\mathcal{K}_{A}(E) \cong B$ (under the map determined by the assignment $\left.\theta_{x, y} \mapsto{ }_{B}\langle x, y\rangle\right)$. As observed in the proof of Theorem 1.6, we have $P_{E}=K_{B} E$, and so $P_{E}$ coincides with the Pedersen submodule of the Hilbert left $B$-module $E$. It is clear that $\left\langle P_{E}, P_{E}\right\rangle_{A}=K_{A}$ and that $P_{E} K_{A}=P_{E}$. (Note that we don't need to take closures here.) Nondegeneracy of $P_{E}$ as a right $K_{A}$-module is also very easy to check: Assume that $e \in P_{E}$ is non-zero. Then $x:=e\langle e, e\rangle_{A}$ is a non-zero element in $e K_{A}$, because $\langle x, x\rangle_{A}=\left(\langle e, e\rangle_{A}\right)^{3} \neq 0$. It follows that $P_{E} \in \operatorname{Mod}-K_{A}$. All the properties also hold on the left because $P_{E}$ is the Pedersen submodule of the Hilbert left $B$-module $E$. In particular, $P_{E} \in K_{B}-M o d$, and we obtain that $P_{E}$ is a full inner product $K_{B}-K_{A}$-bimodule. 
Theorem 2.4. Let $A$ and $B$ be $C^{*}$-algebras. Then $A$ and $B$ are strongly Morita equivalent if and only if $K_{A}$ and $K_{B}$ are Morita $*$-equivalent.

Proof. Assume that $A$ and $B$ are strongly Morita equivalent. Then there is a full Hilbert $B-A$-bimodule $E$ such that $B \cong \mathcal{K}_{A}(E)$ and $A \cong \mathcal{K}_{B}(\widetilde{E})$. Here, $\widetilde{E}$ denotes the Hilbert $A-B$ module which coincides with $E$ as complex Banach space, but with module operations given by $\widetilde{x} b=\widetilde{b^{*} x}$ and $a \widetilde{x}=\widetilde{x a^{*}}$ for $x \in E, a \in A$ and $b \in B$. By Lemma 2.3, the Pedersen submodule of $E_{A}$ coincides with the Pedersen submodule of ${ }_{B} E$. Denote by $P_{E}$ this dense subbimodule of $E$. By Lemma 2.3, the restrictions to $P_{E}$ of the $A$-valued and $B$-valued inner products on $E$ give $P_{E}$ the structure of a full inner product $K_{B}-K_{A}$-bimodule, and $K_{B}$ is $*$-isomorphic to $\mathcal{F}_{A}\left(P_{E}\right)$ by Theorem 1.6. It follows that $K_{A}$ and $K_{B}$ are Morita $*$-equivalent.

Conversely, assume that $K_{A}$ and $K_{B}$ are Morita *-equivalent. By Definition 2.2, this means that there exist $E \in \operatorname{Mod}-K_{A}$ and a map $\langle\cdot, \cdot\rangle: E \times E \rightarrow K_{A}$ such that:

(i) $\langle x, y+z\rangle=\langle x, y\rangle+\langle x, z\rangle$ for all $x, y, z \in E$,

(ii) $\langle x, y r\rangle=\langle x, y\rangle r \quad\left(x, y \in E, r \in K_{A}\right)$,

(iii) $\langle x, y\rangle=\langle y, x\rangle^{*} \quad(x, y \in E)$,

(iv) $\langle x, E\rangle=0$ implies $x=0$,

(v) $\langle E, E\rangle=K_{A}$,

(vi) $K_{B}$ is $*$-isomorphic to $\mathcal{F}_{A}(E)$.

By (vi), the algebra $\mathcal{F}_{A}(E)$, with its natural involution and the norm induced by the norm of $K_{B}$, becomes a pre- $C^{*}$-algebra, and in fact a pre- $C^{*}$-algebra of a very special nature. For example, it follows from [17, 5.6.2] that, given a finite subset of $\mathcal{F}_{A}(E)$, the hereditary $C^{*}$-subalgebra generated by this subset in the completion of $\mathcal{F}_{A}(E)$ is in fact contained in $\mathcal{F}_{A}(E)$.

We want to prove the following additional property:

(vii) $\langle x, x\rangle \geq 0$ for all $x \in E$. Moreover, $\langle x, x\rangle=0$ implies $x=0$.

We first prove the second part of (vii). Assume that $x$ is a nonzero element of $E$. Then $\theta_{x, x}$ must be nonzero. Otherwise, we would have, for all $y \in E$,

$$
0=\left\langle y, \theta_{x, x}(y)\right\rangle=\langle y, x\langle x, y\rangle\rangle=\langle y, x\rangle\langle x, y\rangle=\langle x, y\rangle^{*}\langle x, y\rangle .
$$

This would imply $\langle x, y\rangle=0$ for all $y \in E$ and thus $x=0$ by (iv). Now since $\theta_{x, x}$ belongs to a pre- $C^{*}$-algebra and $\theta_{x, x}^{*}=\theta_{x, x}$, we get $0 \neq\left(\theta_{x, x}\right)^{*}\left(\theta_{x, x}\right)=\theta_{x\langle x, x\rangle, x}$ and consequently $\langle x, x\rangle \neq 0$.

In order to prove the first assertion in (vii), we first prove that for all $x \in E$ and $a \in\left(K_{A}\right)^{+}$, the operator $T:=\theta_{x a, x}$ is positive in the pre- $C^{*}$-algebra $\mathcal{F}_{A}(E)$. First note that $T=T^{*}$, and that for all $z \in E$ we have

$$
\begin{aligned}
\langle T(z), z\rangle & =\left\langle\theta_{x a, x}(z), z\right\rangle=\langle x a\langle x, z\rangle, z\rangle \\
& =\langle z, x\rangle a\langle x, z\rangle \geq 0 .
\end{aligned}
$$

Since the hereditary $C^{*}$-subalgebra of the completion of $\mathcal{F}_{A}(E)$ generated by $T$ is already contained in $\mathcal{F}_{A}(E)([17,5.6 .2])$ we can write $T=T_{1}-T_{2}$ for some $T_{1}, T_{2} \in \mathcal{F}_{A}(E)^{+}$with $T_{1} T_{2}=0$. So we have

$$
\begin{aligned}
0 \leq\left\langle T\left(T_{2}(z)\right), T_{2}(z)\right\rangle & =\left\langle\left(T_{1}-T_{2}\right)\left(T_{2}(z)\right), T_{2}(z)\right\rangle=-\left\langle T_{2}^{3}(z), z\right\rangle \\
& =-\left\langle T_{2}^{3 / 2}(z), T_{2}^{3 / 2}(z)\right\rangle \leq 0 .
\end{aligned}
$$

Therefore, $T_{2}^{3 / 2}=0$ and so $T_{2}=0$. We conclude that $T=T_{1} \geq 0$. 
For $x \in E$ write $a=\langle x, x\rangle$. Then $a$ is a self-adjoint element of $K_{A}$ and so another application of [17, 5.6.2] gives that we can write $a=a_{1}-a_{2}$ with $a_{1}, a_{2} \in\left(K_{A}\right)^{+}$ and $a_{1} a_{2}=0$. We compute

$$
\begin{aligned}
0 \leq \theta_{x a_{2}, x}^{*}\left(\theta_{x, x}^{*} \theta_{x, x}\right) \theta_{x a_{2}, x} & =\theta_{x, x a_{2}} \theta_{x a, x} \theta_{x a_{2}, x} \\
& =\theta_{x\left\langle x a_{2}, x a\right\rangle\left\langle x, x a_{2}\right\rangle, x} \\
& =\theta_{x a_{2}\left(a_{1}-a_{2}\right)^{3} a_{2}, x} \\
& =-\theta_{x a_{2}^{5}, x} .
\end{aligned}
$$

But, being $a_{2}$ positive, $\theta_{x a_{2}^{5}, x}$ is also positive as showed before, and so we conclude that $\theta_{x a_{2}^{5}, x}=0$ and consequently, $x a_{2}^{5 / 2}=0$. But then $0=\left\langle x a_{2}^{5 / 2}, x a_{2}^{5 / 2}\right\rangle=-a_{2}^{6}$, which implies $a_{2}=0$. We conclude that $a=a_{1} \geq 0$, and (vii) is proved.

We have proved that $E$ is a full inner product right $K_{A}$-module in the sense of Definition 1.1. Therefore we can complete $E$ to obtain a full Hilbert $A$-module $\bar{E}$. Since $E$ is dense in $\bar{E}$, we see that $\mathcal{F}_{A}(E)$ is a dense $*$-subalgebra of the $C^{*}$-algebra $\mathcal{K}_{A}(E)$. It follows that $B$ is *-isomorphic to $\mathcal{K}_{A}(E)$ and therefore $A$ and $B$ are strongly Morita equivalent $C^{*}$-algebras.

We don't know whether Morita equivalence between $K_{A}$ and $K_{B}$ implies Morita *-equivalence of $K_{A}$ and $K_{B}$ (the converse is obvious).

\section{REFERENCES}

[1] G. Abrams, Morita equivalence for rings with local units, Comm. in Algebra 11(8) (1983), 801-837. MR 85b:16037

[2] F.W. Anderson and K.R. Fuller, Rings and Categories of Modules (2nd ed.), Springer-Verlag, New York, 1992. MR 94i:16001

[3] P.N. Anh and L. Marki, Morita equivalence for rings without identity, Tsukuba J. Math. 11 (1987), 1-16. MR 88h:16054

[4] P. Ara, Morita equivalence for rings with involution, Algebras and Representation Theory 2 (1999), 227-247. CMP 2000:02

[5] W. Beer, On Morita equivalence of nuclear $C^{*}$-algebras, J. Pure Applied Algebra 26 (1982), 249-267. MR 84d:46096

[6] D. Blecher, On Morita's Fundamental Theorem for $C^{*}$-algebras, to appear in Math. Scand.

[7] L.G. Brown, P. Green and M.A. Rieffel, Stable isomorphism and strong Morita equivalence of $C^{*}$-algebras, Pacific J. Math. 71 (1977), 349-363. MR 57:3866

[8] L.G. Brown, J.A. Mingo and N.T. Shen, Quasi-multipliers and embeddings of Hilbert $C^{*}$ bimodules, Can. J. Math. 46 (1994), 1150-1174. MR 95k:46091

[9] J.L. Garcia and J.J. Simon, Morita equivalence for idempotent rings, J. Pure Applied Algebra 76 (1991), 39-56. MR 93b:16010

[10] P. Green, Local structure of twisted covariant algebras, Acta Math. 140 (1978), 191-250. MR 58:12376

[11] G.G. Kasparov, Hilbert $C^{*}-$ modules: Theorems of Stinespring and Voiculescu, J. Operator Theory 4 (1980), 133-150. MR 82b:46074

[12] S. Kyuno, Equivalence of module categories, Math J. Okayama Univ. 28 (1986), 147-150. MR 88e:16065

[13] E.C. Lance, Hilbert $C^{*}$-modules, Cambridge Univ. Press, LMS Lecture Note Series 210 Cambridge, 1995. MR 96k:46100

[14] A.J. Lazar and D.C. Taylor, Multipliers of Pedersen's ideal, Memoirs Amer. Math. Soc. 5 (1976). MR 54:944

[15] N. Nobusawa, $\Gamma$-rings and Morita equivalence of rings, Math. J. Okayama Univ. 26 (1984), 151-156. MR 86d:16048

[16] M. Parvathi and A. Ramakrishna Rao, Morita equivalence for a larger class of rings, Publ. Math. Debrecen 35 (1988), 65-71. [MR 89m:16085]

[17] G.K. Pedersen, $C^{*}$-algebras and their automorphism groups, Academic Press, London, 1979. MR 81e: 46037 
[18] N.C. Phillips, A new approach to the multipliers of Pedersen's ideal, Proc. Amer. Math. Soc. 104 (1988), 861-867. MR 89h:46081

[19] M.A. Rieffel, Induced representations of $C^{*}$-algebras, Advances in Math. 13 (1974), 176-257. MR 50:5489

[20] M.A. Rieffel, Morita equivalence for $C^{*}$-algebras and $W^{*}$-algebras, J. Pure Applied Algebra 5 (1974), 51-96. MR 51:3912

[21] M.A. Rieffel, Morita equivalence for operator algebras, Proc. Sym. Pure Math. 38, 285-298, Amer. Math. Soc., Providence, R.I., 1982. MR 84k:46045

[22] B. Stenstrom, Rings of Quotients, Springer-Verlag, 1975. MR 52:10782

Departament de Matemàtiques, Universitat Autònoma de Barcelona, 08193 Bellaterra (BARCElona), Spain

E-mail address: para@mat.uab.es 